\title{
Anti-Inflammatory Effects of Vardenafil Against Cholestatic Liver Damage in Mice: a Mechanistic Study
}

\author{
Dina S. El-Agamy ${ }^{a, b} \quad$ Hamdi H. Almaramhyc Nishat Ahmed ${ }^{a} \quad$ Bsmah Bojan $^{d}$ \\ Waad D. Alrohily ${ }^{d}$ Mohamed A. Elkablawye,f
}

aDepartment of Pharmacology and Toxicology, College of Pharmacy, Taibah University, Al-Madinah Al-Munawwarah, Saudi Arabia, 'bepartment of Pharmacology and Toxicology, Faculty of Pharmacy, Mansoura University, Mansoura, Egypt, 'Department of Surgery, College of Medicine, Taibah University, Al-Madinah Al-Munawwarah, dDepartment of Clinical Pharmacy, College of Pharmacy, Taibah University, Al-Madinah Al-Munawwarah, eDepartment of Pathology, College of Medicine, Taibah University, Al-Madinah Al-Munawwarah, Saudi Arabia; ${ }^{f}$ Department of pathology, Faculty of Medicine, Menoufia University, Menoufia, Egypt

\section{Key Words}

Lithocholic acid • Hepatotoxicity • Vardenafil • Nrf2 • NLRP3 inflammasome

\begin{abstract}
Background/Aims: Phosphodiesterase-5 inhibitors have beneficial effects in multiple liver diseases possibly through the reduction of oxidative stress and inflammatory response. However, these effects have not yet been examined in cholestatic liver dysfunction. Hence, this study aimed to explore the ability of vardenafil, a known phosphodiesterase- 5 inhibitor, to repress lithocholic acid (LCA)-induced cholestatic liver injury and investigate the possible molecular pathways. Methods: Male Swiss albino mice were treated with LCA $(0.125 \mathrm{mg} / \mathrm{g})$ twice daily for 7 days to induce cholestatic liver damage. Vardenafil was administered 3 days before and throughout the administration of LCA. Serum markers of hepatotoxicity and hepatic nitrooxidative stress along with antioxidant parameters were measured, and the histopathology of liver tissues was assessed. The expression of nuclear factor erythroid 2-related factor 2 (Nrf2) and its target genes was examined using PCR. The activation of nuclear factor kappa-B (NF$\mathrm{KB}$ ) and the levels of inflammatory cytokines were determined. NLRP3 inflammasome and its components were studied by PCR and western blot. Results: LCA induced marked cholestatic liver damage as demonstrated by increased serum transaminases, alkaline phosphatase $(A L P)$, lactate dehydrogenase (LDH), bilirubin, and bile acids. Examination of liver specimens confirmed the biochemical results. Nitro-oxidative stress parameters were significantly elevated along with reduced antioxidant capacity in hepatic tissue following LCA administration. LCA suppressed Nrf2 and its target genes and decreased the mRNA expression and binding capacity of Nrf2 as well as the mRNA expression of GCLm, GCLc, Nqo1, and HO-1. Additionally, LCA enhanced the activation of NF-KB, which was accompanied by elevations of inflammatory
\end{abstract}


cytokines. Importantly, LCA induced the activation of NLRP3 inflammasome. LCA increased the expression of NLRP3, ASC, caspase- 1 , and IL-1 $\beta$ genes and proteins in hepatic tissue. The activities of IL-1 $\beta$ and caspase- 1 were increased in the LCA group. Interestingly, vardenafil ameliorated LCA-induced hepatic injury and alleviated all biochemical, histopathological, and inflammatory parameters. Conclusions: These data elucidated the effects of Nrf2 inhibition and NLRP3 inflammasome activation in LCA-induced liver injury. The hepatoprotective activity of vardenafil in LCA-induced cholestatic damage may result from the drug's ability to activate Nrf2 signaling and prevent the activation of NLRP3, which could suppress the inflammatory responses in hepatic tissue. Thus, vardenafil can be considered a novel anti-inflammatory remedy for cholestatic liver damage.

(C) 2018 The Author(s)

Published by S. Karger AG, Basel

\section{Introduction}

Cholestasis is a pathological state characterized by impaired bile flow from the liver. The condition occurs in many diseases such as biliary obstruction, biliary atresia, intrahepatic cholestasis, and cholangiocarcinoma. An accumulation of highly toxic bile acids in the hepatocytes leads to cytotoxicity and cholestatic liver injury. Persistent cholestatic liver damage results in marked inflammatory reactions that can eventually lead to the activation and proliferation of hepatic stellate cells, overproduction of matrix metalloproteinases, synthesis of extracellular matrix, liver fibrosis, cirrhosis, and even death [1-3].

Nuclear factor-erythroid 2 related factor 2 (Nrf2) is a transcription factor that plays an important role in the amelioration of various inflammatory diseases, including cholestatic hepatic injury [4-5]. Under normal conditions, Nrf2 exists as heterodimers with the cytosolic repressor Keap1. Stress conditions activate Nrf2 release and its translocation into the nucleus where it induces the expression of antioxidant cytoprotective genes such as superoxide dismutase (SOD), the glutamate-cysteine ligase modifier (GCLm), the catalytic (GCLc) subunit, NAD(P)H: quinone oxidoreductase (Nqo1), and heme oxygenase-1 (HO-1) [6]. Previous studies have shown that the activation of Nrf2 signaling along with its target genes provide protection against cholestatic liver injury induced by either bile duct ligation or the administration of alpha-naphthyl isothiocyanate [4-5, 7].

The NOD-like receptor 3 (NLRP3) inflammasome is a well-defined cytoplasmic protein complex that can be activated by various stimuli. This multimolecular complex consists of NLRP3 protein, apoptosis-associated speck-like protein containing a caspase-activating recruitment domain (ASC), and caspase-1. The role of NLRP3 inflammasome activation in the progression of hepatic inflammation and fibrosis is receiving widespread attention [8-10]. The activation of NLRP3 inflammasome results in the secretion of inflammatory cytokines such as interleukin-1 $\beta$ (IL-1 $\beta$ ) and IL-18. Moreover, IL-1 $\beta$ has been shown to be elevated in cases of bile duct ligation-induced cholestatic liver injury in mice. It promotes the recruitment of inflammatory cells into the hepatic tissue and induces fibrogenesis $[11,12]$. However, the exact role of NLRP3 inflammasome in mediating cholestatic liver damage is not completely understood.

Phosphodiesterase type 5 (PDE-5) inhibitors are administered for the treatment of erectile dysfunction (ED) and pulmonary arterial hypertension. These agents inhibit the breakdown of cyclic guanosine monophosphate (cGMP) and potentiate its activity leading to smooth muscle relaxation and vasodilation in the pulmonary vasculature and corpus cavernosum [13]. In addition to these beneficial effects, these agents have pleiotropic effects that protect hepatic tissue. Sildenafil, a selective PDE-5 inhibitor, ameliorates the development of thioacetamide-induced hepatic fibrosis by inhibiting the release of profibrogenic inflammatory cytokines [14]. Furthermore, sildenafil exerts hepatoprotective effects in hepatic ischemia reperfusion injury through the modulation of ICAM and apoptosis [15]. More recently, vardenafil, another selective PDE-5 inhibitor, has been shown to offer protective effects in cystic fibrosis [16] and experimentally induced hepatitis by suppressing oxidative stress and inhibiting the activation of nuclear factor kappa-B (NF- $\kappa \mathrm{B})$ a [17]. 
El-Agamy et al.: Vardenafil and Cholestatic Liver

However, the effects of PDE-5 inhibitors in cholestatic liver disease have not yet been discussed. Hence, the current investigation was designed to explore the mechanism and extent of NLRP3 inflammasome activation in lithocholic acid (LCA)-induced cholestatic hepatic damage and assess whether vardenafil has hepatoprotective effects in LCA-induced cholestasis.

\section{Materials and Methods}

Chemicals and kits

LCA and vardenafil hydrochloride trihydrate were purchased from Sigma-Aldrich (St. Louis, MO, USA). LCA was dissolved in DMSO while vardenafil was prepared in saline. Ketamine (Tekam ampoules) was generously provided by the Medical Centre of Taibah University (Al-Madinah Al-Munawwarah, Saudi Arabia). Alanine aminotransferase (ALT), aspartate aminotransferase (AST), alkaline phosphatase (ALP), lactate dehydrogenase (LDH), and bilirubin kits were purchased from Human (Wiesbaden, Germany). Total bile acid (TBA) and caspase-1 assay kits were bought from BioVision Inc. (Milpitas, CA, USA). Malondialdehyde (MDA), reduced glutathione (GSH), SOD, and total antioxidant capacity (TAC) kits were purchased from Bio-diagnostic Co. (Giza, Egypt). ELISA kits for 4-hydroxynonenal (4-HNE) were purchased from MyBioSource Inc. (San Diego, CA, USA), while those for nitrite/nitrate (NOx), tumor necrosis factor- $\alpha$ (TNF- $\alpha$ ), interleukine-1 $\beta$ (IL-1 $\beta$ ), and IL-6 were purchased from R\&D Systems (Minneapolis, MN, USA). To estimate the expression of Nrf2, TransAM Nrf2 ELISA kit was obtained from Active Motif (Carlsbad, CA, USA). Nuclear extraction and NF-kB p65 activation kits were purchased from Abcam (Cambridge, MA, USA).

\section{LCA-induced cholestatic liver damage}

Male Swiss albino mice weighing 20-25 g were obtained from Experimental Animal Facility, College of Pharmacy, Taibah University. The study was conducted according to the protocol of the Research Ethics Committee, Taibah University, which adheres to the "Principles of Laboratory Animals Care" (NIH publication). Animals had access to standard chow and water under usual conditions of humidity, temperature, and light/dark cycle.

Cholestatic liver injury was induced by LCA administration as previously described [18]. In brief, mice were randomly assigned into four experimental groups each comprising 10 mice. The groups were treated as follows: Control group animals received normal saline; LCA group animals received intraperitoneal injections of LCA $(0.125 \mathrm{mg} / \mathrm{g})$ twice daily for 7 days from days 4 to 10 ; and LCA + vardenafil 0.15 group and LCA + vardenafil 0.3 group animals received a single intraperitoneal injection of vardenafil at doses of $0.15 \mathrm{mg} / \mathrm{kg}$ and $0.3 \mathrm{mg} / \mathrm{kg}$, respectively, once daily for 10 days along with LCA (from day 4 to day 10). All mice were sacrificed by ketamine anesthesia at the end of the treatment period. The blood was collected and then the serum was immediately separated. Fresh liver tissues were isolated and weighed. Small pieces of the liver tissues were weighed and homogenized in RIPA buffer. After centrifugation, the supernatants were obtained and stored for further analysis. Part of the liver was stored at $-80^{\circ} \mathrm{C}$ for further experiments. The right lobe of the liver was fixed in $10 \%$ neutral buffered formalin and submitted for pathological assessment.

Measurements of hepatic injury indices

Serum ALT, AST, ALP, LDH, bilirubin (direct and total), and TBA were measured using commercial kits as per the manufacturers' instructions. Colorimetric measurements were performed using a spectrophotometer (Model 1200, UNICO Instruments Co., Dayton, NJ, USA). In addition, TBA content in the supernatants of hepatic homogenates was measured according to the protocol for the kit.

\section{Liver pathological examination}

Fixed hepatic tissues were embedded in paraffin and sliced into 5 - $\mu$ m sections. Liver specimens were stained by hematoxylin-eosin and examined in random order. The histological damage in liver tissues was evaluated as described previously [19]. Briefly, the extent of hepatic injury was scored from 0 to 4 based on the proportion of pathological changes in the field.

\section{Biochemical measurements}

Nitro-oxidative stress and antioxidant status parameters. Supernatants of liver homogenates were used to measure 4-HNE, MDA, GSH, SOD, TAC, and NOx based on the kit protocols. 


\section{Cellular Physiology Cell Physiol Biochem 2018;47:523-534 \begin{tabular}{l|l} 
and Biochemistry Published online:May 24, 2018 & $\begin{array}{l}\text { D) } 2018 \text { The Author(s). Published by S. Karger AG, Basel } \\
\text { www.karger.com/cpb }\end{array}$ \\
\hline
\end{tabular}}

El-Agamy et al.: Vardenafil and Cholestatic Liver

Nrf2 binding activity. The binding activity of Nrf2 was estimated in the hepatic nuclear extract according to the guidelines. Briefly, the active form of Nrf2 bound specifically to the immobilized oligonucleotide containing the ARE consensus binding site. A secondary HRP-conjugated antibody was added and the colored product was quantified by spectrophotometry at $450 \mathrm{~nm}$.

$N F-\kappa B$ p 65 activation. Activation of NF- $\kappa B$ p 65 was determined in the hepatic nuclear extract according to the kit protocol. Results were represented as OD at $450 \mathrm{~nm}$.

Cytokines. Serum level of IL-1 $\beta$ and levels of TNF- $\alpha$, IL-1 $1 \beta$, IL-6, and caspase- 1 in the supernatants of liver homogenates were measured according to the protocols provided by the manufacturer.

\section{Real-time PCR (RT-PCR) analysis}

Gene expression of Nrf2 and NLPR3 inflammasome contents in the hepatic tissue were estimated. Separation of total RNA was performed using RNeasy Mini kit (Qiagen, Valencia, CA, USA). The purity of the isolated RNA was quantified using the ratio A260/A280. Reverse transcription of RNA to CDNA was performed with the Quantitect Reverse Transcription Kit (Qiagen). Finally, RT-PCR was performed using SYBR Green (Qiagen) according to the manufacturer's protocol. The following primers were used: Nrf2 (mouse) 5'-CCTCGCTGGAAAAAGAAGTG-3' (sense), 5'-GGAGAGGATGCTGCTGAAAG-3' (antisense); GCLm (Mouse) $5^{\prime}$-AGGAGCTTCGGGACTGTATCC-3' (sense)' 5'-GGGACATGGTGCATTCCAAAA-3' (antisense); GCLc (mouse) $5^{\prime}$-TTCCTGGACTGATCCCAATTCTG-3' (sense), 5'- CTCATCCATCTGGCAACTGTCATTA-3' (antisense); Nqo1 (mouse) 5'-ATGGGAGGTGGTCGAATCTGA-3' (sense), 5'-GCCTTCCTTATACGCCAGAGATG-3' (antisense); HO-1 (mouse) 5'-AAGCCGAGAATGCTGAGTTCA-3' (sense), 5' ${ }^{\prime}$-GCCGTGTAGATATGGTACAAGGA-3' (antisense); IL-1 $\beta$ (mouse) 5'-TGGACGGACCCCAAAAGATG-3' (sense), 5'-AGAAGGTGCTCATGTCCTCA-3' (antisense); Caspase-1 (mouse) $5^{\prime}$ - AGGAATTCTGGAGCTTCAATCAG-3' (sense), $5^{\prime}$-TGGAAATGTGCCATCTTCTTT-3' (antisense); ASC (mouse) 5'-ACAGAAGTGGACGGAGTGCT-3' (sense), 5'- CTCCAGGTCCATCACCAAGT-3' (antisense); NLRP3 (mouse) 5'-GCTCCAACCATTCTCTGACC-3' (sense), 5'-AAGTAAGGCCGGAATTCACC-3' (antisense); and $\beta$-actin (mouse) $5^{\prime}$ - TCTACGAGGGCTATGCTCTCC-3' (sense), 5'-GGATGCCACAGGATTCCATAC-3' (antisense). The expression levels of different genes were normalized to $\beta$-actin as a housekeeping gene. The relative expression of each gene was calculated using the $\Delta \Delta \mathrm{Ct}$ method. Rotor-Gene Q Software 2.1 (Qiagen) was used to analyze the data.

Western blot analysis

To determine the protein expression levels, total protein was extracted from hepatic tissue and processed as previously described [19]. In brief, the membranes were blocked with 5\% non-fat milk in buffer (20 mM Tris $\mathrm{HCl}, \mathrm{pH} 7.4,135 \mathrm{mM} \mathrm{NaCl}, 0.1 \%$ Tween) and incubated overnight at $4{ }^{\circ} \mathrm{C}$ with primary antibodies against IL-1 $\beta$ (1:1000; Cell Signaling Technology, Danvers, MA, USA), caspase-1 (1:200; Santa Cruz Biotechnology, Dallas, TX, USA), ASC (1:500; Biorbyt Ltd., Cambridge, UK), NLRP3 (1:1000; Novus Biologicals, Littleton, CO, USA), and $\beta$-actin (1:5000; Santa Cruz Biotechnology). The membranes were then incubated with secondary antibodies: anti-mouse $(1: 200,1 \mathrm{~h}$, at room temperature) conjugated with horseradish peroxidase (Santa Cruz Biotechnology).

\section{Immunohistochemical analysis}

Immunohistochemical (IHC) analysis of hepatic tissue obtained from each animal was performed as previously described [19]. Briefly, tissue mini-arrays (TmA) were constructed from paraffin liver blocks. IHC staining for caspase-1 was performed automatically using Ventana Bench Mark XT system (Ventana Medical Systems, Tucson, AZ, USA). IHC staining was carried out using primary antibodies against anti caspase-1 antibody (1:100).

\section{Statistical analysis}

Data analysis was performed by one-way analysis of variance followed by Tukey-Kramer multiple comparison post-hoc test. Histopathological score analysis was performed by a non-parametric KruskalWallis test followed by Dunn's multiple comparison post-hoc test. The values are presented as the mean \pm standard error of the mean for each group. A P value $<0.05$ was considered statistically significant. 


\section{Results}

\section{Effect of vardenafil on LCA-induced cholestatic liver injury}

As presented in Fig. 1, the administration of LCA resulted in hepatic injury demonstrated by significant elevations $(\mathrm{P}<0.001)$ in serum transaminases, ALP, and LDH compared with the control. Furthermore, LCA induced remarkable increases in the levels of bilirubin and TBA, which are indices of cholestasis. Vardenafil co-treatment dose-dependently reversed the LCA-induced increases in the biochemical parameters of cholestatic hepatic damage. Notably, histopathological assessments of the liver specimens confirmed the biochemical results. LCA induced marked hepatic damage in the form of local coagulative necrosis, degeneration, infiltration by inflammatory cells, and proliferation of the bile ducts (Fig. 1E). On the other hand, liver specimens in the vardenafil + LCA groups showed clear limitation of LCA-induced hepatic injury in a dose-dependent manner (Fig. 1F). The hepatic tissues of mice receiving high doses of vardenafil along with LCA showed almost normal hepatic architecture.

\section{Effect of vardenafil on nitrosative oxidative stress parameters}

LCA induced a significant increase $(\mathrm{P}<0.001)$ in lipid peroxidation in the hepatic tissues, indicated by elevations in the levels of lipid peroxidation deleterious products, namely,

Fig. 1. Effects of vardenafil on the biochemical and histopathological parameters of LCA-induced cholestatic liver injury in mice. (A) Serum transaminases ALT and AST. (B) Serum ALP and LDH. (C) Serum total and direct bilirubin. (D) Serum and liver TBA content. (E) Representative liver sections stained with hematoxylin-eosin $(\times 400)$ showing normal hepatic histology in control mice and proliferation of the bile ducts (lightning bolts), hepatic necrosis and degeneration (arrows), apoptosis (arrow heads), and infiltration of inflammatory cells (stars) in a liver specimen of the LCA group. The vardenafil 0.15 + LCA group showed mild bile duct proliferation and mild infiltration by inflammatory cells. Vardenafil 0.3 + LCA group had normal hepatocytes and histological architecture. (F) Histopathological score. Data are presented as the mean \pm standard error of the mean (SEM) $(\mathrm{n}=10) .{ }^{*} \mathrm{P}<0.05,{ }^{* *} \mathrm{P}<0.01$, ${ }^{* * *} \mathrm{P}<0.001$ vs. the control; ${ }^{\mathrm{P}}<0.05$, ${ }^{\# \#} \mathrm{P}<0.01$, \#\#\# $\mathrm{P}<0.001$ vs. the LCA group (analysis of variance [ANOVA] followed by Tukey-Kramer multiple comparisons test; Kruskal-Wallis fol-
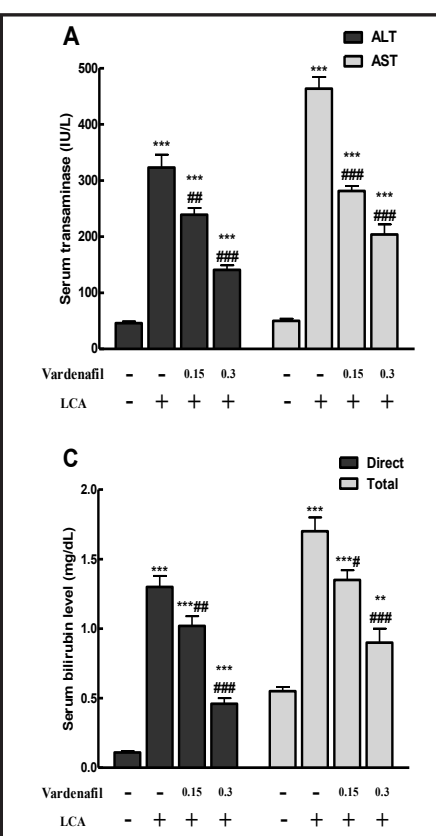

E

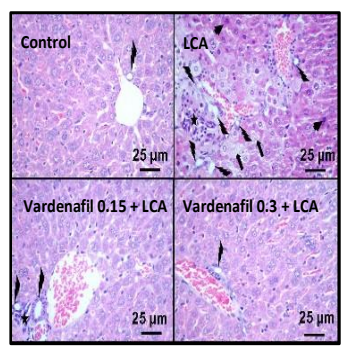

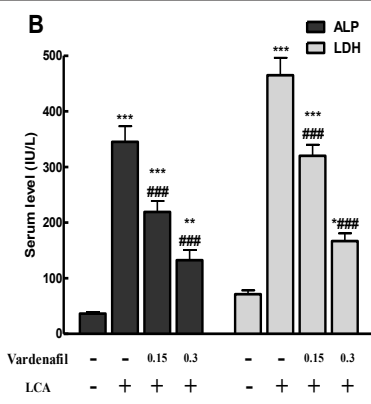

D

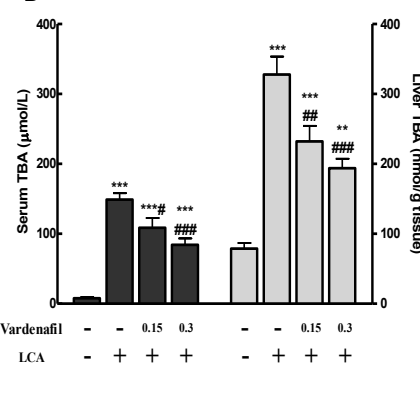

$\mathrm{F}$

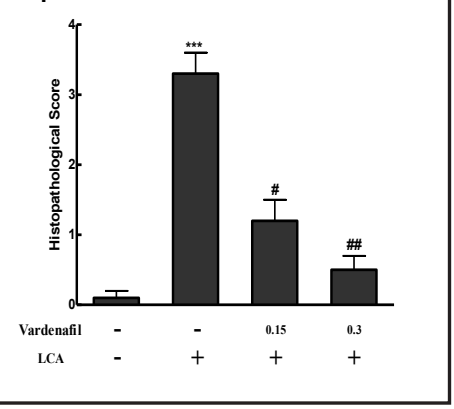
lowed by Dunn's multiple comparisons test was performed for histopathology scores). 


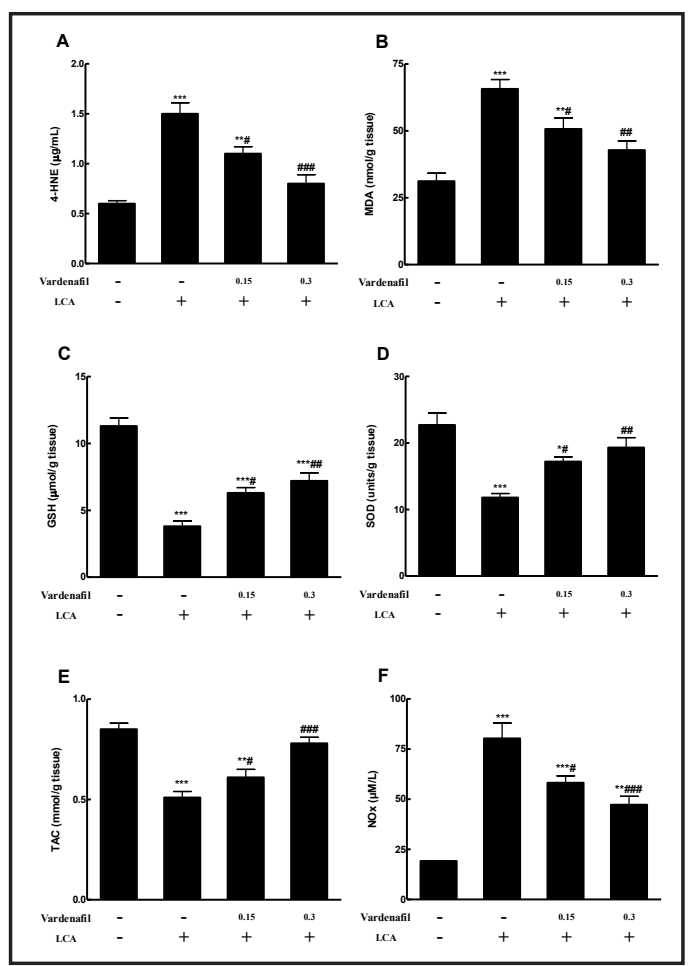

Fig. 2. Effects of vardenafil on LCA-induced nitrooxidative damage in hepatic tissue of mice. Data are presented as the mean \pm SEM $(n=10) .{ }^{*} \mathrm{P}<0.05$, ${ }^{* *} \mathrm{P}<0.01,{ }^{* * *} \mathrm{P}<0.001$ vs. the control; ${ }^{\#} \mathrm{P}<0.05$,

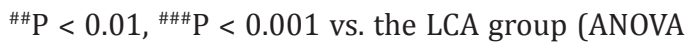
followed by Tukey-Kramer multiple comparisons test).

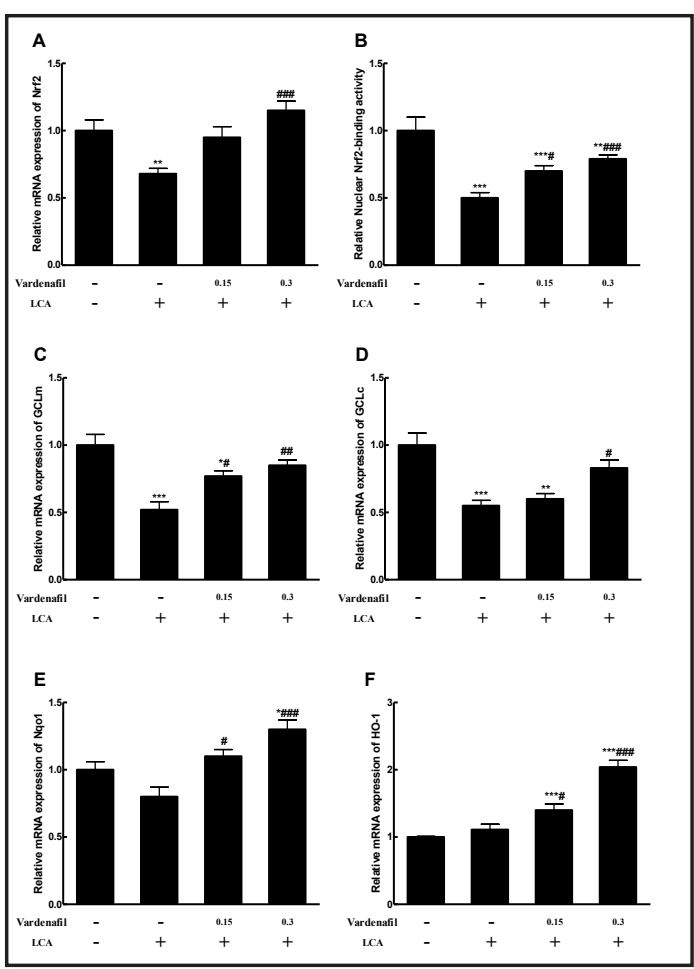

Fig. 3. Effects of vardenafil on Nrf2 pathway in LCAinduced cholestatic liver injury in mice. (A) Relative mRNA expression of Nrf2. (B) Nrf2 binding capacity. Relative mRNA expression of (C) GCLm, (D) GCLc, (E) Nqo1, and (F) HO-1. Data are presented as the mean \pm SEM $(n=10) .{ }^{*} \mathrm{P}<0.05,{ }^{* *} \mathrm{P}<0.01,{ }^{* * *} \mathrm{P}<0.001$ vs. the control; ${ }^{\#}<0.05,{ }^{\# \#} \mathrm{P}<0.01$, ${ }^{\# \# \#} \mathrm{P}<0.001$ vs. the LCA group (ANOVA followed by Tukey-Kramer multiple comparisons test).

4-HNE and MDA (Fig. 2A, B). Furthermore, LCA treatment resulted in depletion of hepatic antioxidants, GSH and SOD, and lower TAC compared with the control group (Fig. 2C-E). LCA administration also led to increased hepatic NOx concentrations $(\mathrm{P}<0.001)$ compared with the control group (Fig. 2F). Concomitant vardenafil use resulted in the restoration of these antioxidants and increased the levels of hepatic TAC, which were associated with significant suppression of nitro-oxidative stress parameters.

\section{Effect of vardenafil on Nrf2 pathway signaling}

As presented in Fig. 3, LCA-treated mice showed significant decreases in the expression of Nrf2, GCLc, and GCLm mRNA and significant decreases in Nrf2 binding activity compared with the control mice (Fig. 3 A-D). On the other hand, vardenafil significantly up-regulated the expression of Nrf2, GCLc, and GCLm mRNA and elevated Nrf2 binding activity compared to the LCA group. The expression levels of Nqo1 and HO-1were not significantly changed in the LCA group compared to the control. However, vardenafil treatment enhanced the mRNA expression compared with the LCA group. This phenomenon may be associated with the enhanced expression of Nrf2 (Fig 3. E \& F)

\section{Effect of vardenafil on the activation of $N F-\kappa B$ and inflammatory mediators}

Compared with the control group, LCA administration resulted in the activation of NF- $\kappa$ B and a significant increase in the levels of inflammatory cytokines TNF- $\alpha$ and IL- 6 in hepatic tissue (Fig. 4). On the other hand, vardenafil treatment significantly inhibited LCA- 
Fig. 4. Effects of vardenafil on the activation of $\mathrm{NF}-\kappa \mathrm{B}$ and levels of inflammatory cytokines (TNF- $\alpha$ and IL-6) in LCA-induced cholestatic injury in mice. Data are presented as the mean \pm SEM $(\mathrm{n}=$ 10). ${ }^{* *} \mathrm{P}<0.01,{ }^{* * *} \mathrm{P}<0.001$ vs. the control; ${ }^{\mathrm{P}}<0.05,{ }^{\# \#} \mathrm{P}<0.01$, , \#\# $\mathrm{P}<$

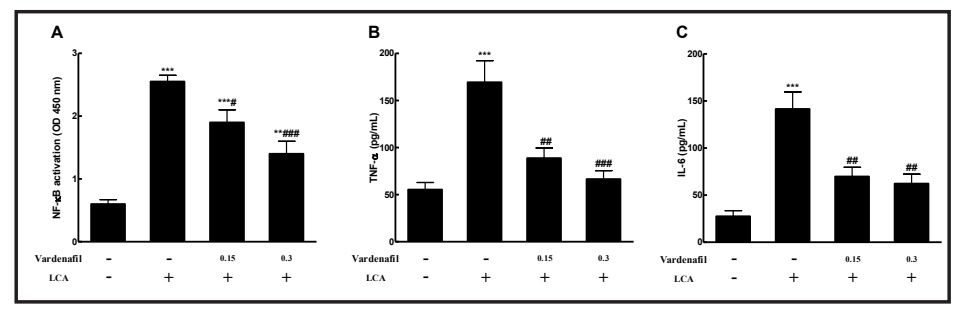
0.001 vs. the LCA group (ANOVA followed by Tukey-Kramer multiple comparisons test).

induced activation of NF- $\mathrm{BB}$ and the overproduction of these cytokines in a dosedependent manner.

\section{Effect of vardenafil on} NLRP3 inflammasome activation

During this step, we examined the effect of LCA administration on the activation of NLRP3 inflammasome and tested whether vardenafil could modulate that effect. The results showed that LCA administration significantly increased the mRNA expression of NLRP3 inflammasome components (ASC, NLRP3, and caspase-1) in addition to IL-1 $\beta$ mRNA compared with the control animals (Fig. 5A, B). In accordance with these data, the expression of pro-IL-1 $\beta$ and pro-caspase-1 proteins as well as their corresponding activated forms (IL-1 $\beta$ and caspase- 1 ), ASC and NLRP3 were significantly enhanced in the LCA group (Fig. 5C). Importantly, the activity of caspase- 1 was significantly elevated in the hepatic tissues of the LCA group and this may be closely associated with the enhanced secretion of IL$1 \beta$ (Fig. 5D,E). On the other hand, vardenafil treatment showed a potent inhibitory

\section{KARGER}

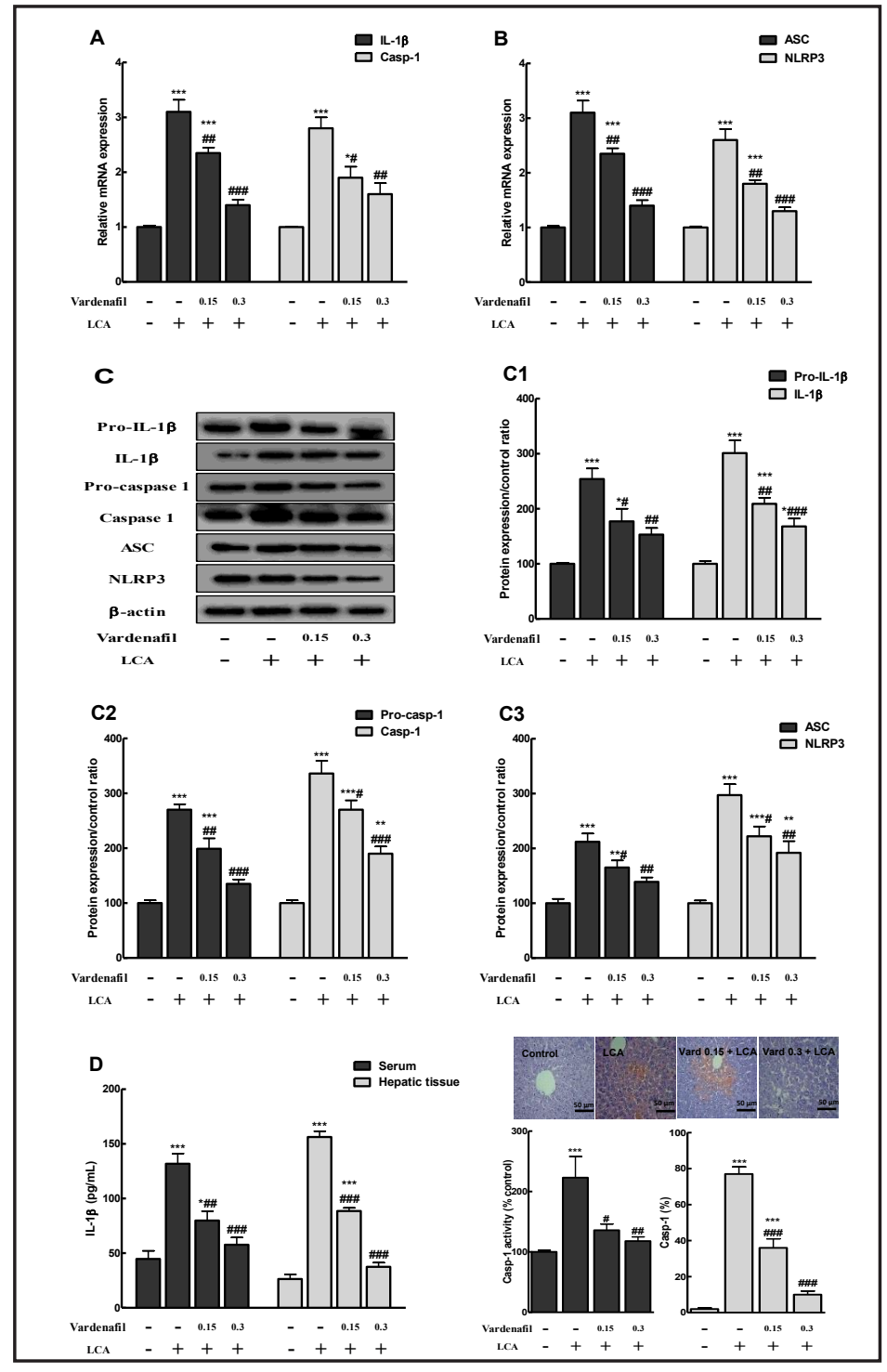

Fig. 5. Effects of vardenafil on NLRP3 inflammasome activation in LCA-induced cholestatic injury in mice. (A \& B) mRNA expression of IL-1 $\beta$, caspase- 1, ASC, and NLRP3 by RT-PCR. (C, C1, C2, \& C3) Protein expression of pro-IL-1 $\beta$, IL-1 $\beta$, pro-caspase1, caspase-1, ASC, and NLRP3. (D) Levels of IL- $\beta$ in serum and hepatic tissue. (E) Caspase-1 activity and the IHC analysis in hepatic tissue $(200 \times)$. Data are presented as the mean \pm SEM $(\mathrm{n}=10) .{ }^{*} \mathrm{P}<0.05,{ }^{* *} \mathrm{P}<0.01,{ }^{* * *} \mathrm{P}<0.001$ vs. the control; " $\mathrm{P}<0.05$, "\# $\mathrm{P}<0.01,{ }^{\# \# \#} \mathrm{P}<0.001$ vs. the LCA group (ANOVA followed by Tukey-Kramer multiple comparisons test). 
effect on the activation of NLRP3 inflammasome. Vardenafil also ameliorated the expression of both the mRNA and proteins of NLRP3 inflammasome components, which led to a dramatic reduction in caspase- 1 activity and consequently IL- $\beta$ secretion.

\section{Discussion}

Cholestasis accompanied with excessive accumulation of bile acids in hepatic tissue can result in cholestatic hepatic fibrosis and cirrhosis [2, 3]. LCA is a monohydroxy hydrophobic bile acid produced in the gut by bacterial reduction of chenodeoxycholic acid [20]. LCA is hepatotoxic and it has been used previously to produce a model of cholestatic liver damage in mice. The accumulation of LCA in cholangioles is associated with biliary obstruction that mimics obstructive cholestasis caused by bile duct ligation [18, 21]. Vardenafil has been reported to possess anti-inflammatory properties. To our knowledge, the key findings of this study are the first to demonstrate the ability of vardenafil to ameliorate LCA-induced cholestatic liver damage through activation of the Nrf2 pathway and the suppression of NLRP3 inflammasome activation.

The biochemical and histopathological results clearly indicated that LCA caused marked cholestatic liver injury. The biochemical data showed significant elevation of hepatocellular damage markers such as serum transaminases, ALP, LDH and other characteristic cholestatic hepatotoxic parameters (bilirubin and TBA) in the LCA group. Distortions and lesions of the hepatic architecture were further confirmed by microscopic examination. However, vardenafil treatment significantly reversed this pathophysiological state and successfully conferred protection against LCA-induced hepatotoxic effects in a dose-dependent manner. This observation is consistent with previous studies that reported the hepatoprotective effects of PDE-5 inhibitors against ischemic liver injury [15], fatty liver [22], and hepatic fibrosis [14]. Furthermore, our lab recently demonstrated the protective effects of vardenafil against autoimmune hepatitis [17].

The release of reactive oxygen and nitrogen species and the associated nitro-oxidative stress play a key role in the pathogenesis of different types of liver injury including cholestatic damage $[5,23]$. In the current study, elevations of lipid peroxidation and reduced antioxidant statuses were observed among the hepatic tissues of LCA-treated animals. Vardenafil treatment significantly inhibited oxidative stress and restored the antioxidant parameters, suggesting that the drug is a powerful inhibitor of LCA-induced hepatic oxidative damage. This finding corresponds with previous investigations that showed the ability of vardenafil to suppress nitro-oxidative damage in the aortic endothelium and rat ovary [24, 25]. Additionally, other PDE-5 inhibitors have been reported to have potential antioxidative effects in other models of oxidative injury [26-29].

Previous reports have clarified the role of Nrf2 as a primary cellular defense pathway. Nrf2 can regulate the expression and activation of variety of enzymes that counteract oxidative stress and promote cell survival $[30,31]$. In addition, studies have shown that the activation of Nrf2 can safeguard against cholestasis caused by bile duct ligation and alphanaphthyl isocyanate models via the induction of many anti-oxidative genes [5, 32, 33]. The induction of HO-1 can confer hepatoprotection in various models of hepatic injuries [34, 35]. The present data corresponded with these previous reports. LCA administration decreased the expression of Nrf2, GCLc, and GCLm mRNA and the binding capacity of Nrf2. Meanwhile, LCA did not induce significant alterations of Nqo1and HO-1 mRNA levels. Interestingly, vardenafil administration significantly enhanced the expression of Nrf2 mRNA along with its downstream genes GCLc, GCLm, Nqo1, and HO-1. Moreover, vardenafil augmented the binding capacity of Nrf2and the activation of Nrf2/HO-1 may partly explain the increased activity of SOD in vardenafil-treated groups. These results are consistent with previous reports that showed the ability of the PDE-5 inhibitor sildenafil to activate the Nrf2/HO-1 signaling pathway [36,37]. Similarly, Liu et al [38]. demonstrated the ability of sildenafil 
to induce the expression of HO-1. Taken together, we presumed that the hepatoprotective effect of vardenafil against LCA-induced cholestatic damage might be partly mediated via the activation of Nrf2 and its target genes.

Since there is strong crosstalk between the activation of Nrf2 and attenuation of the inflammatory NF- $\kappa B$ pathway, it was essential to evaluate the activation of NF- $\kappa B$ and its inflammatory cascade in hepatic tissue. The nuclear transcription factor, NF- $\kappa \mathrm{B}$ is activated by various factors such as oxidative stress and bile acids. It is transferred into the nucleus, where it induces the production of a series of proinflammatory cytokines. NF- $\kappa B$ was shown to be involved in the mediation of the inflammatory response in alpha-naphthyl isothiocyanateinduced cholestatic liver injury [39]. TNF- $\alpha$ is considered one of the basic cytokines involved in the mediation and even control of liver injury through the induction of other cytokines and subsequent amplification of the inflammatory response. Cholestatic liver damage has been reported to be associated with the marked release of inflammatory cytokines such as TNF- $\alpha$, NOx, IL-6, and IL-1 $\beta$, which can cause hepatic injury $[40,41]$. Our data showed that LCA induced the activation of NF- $\kappa B$ and markedly increased the release of inflammatory cytokines, and these effects were greatly reduced by vardenafil treatment. These findings are consistent with previous investigations that showed the ability of vardenafil to suppress $\mathrm{NF}-\kappa \mathrm{B}$ activation and subsequently the release of inflammatory cytokines in autoimmune hepatitis [17] and lung inflammation [42].

Previous reports have elucidated the pivotal role of NLRP3 inflammasome activation as a trigger of the inflammatory response and promoter of hepatic inflammation and fibrosis [8]. The activation of NLRP3 inflammasome is linked to the excessive production of reactive oxygen species and increased oxidative stress [43]. Additionally, NLRP3 can be activated by various exogenous and endogenous activators such as uric acid, silica, bacteria and toxins [44-45]. Activated NLRP3 inflammasome induces the release of active caspase- 1 from its precursor (procaspase-1), which in turn stimulates the release of mature IL-1 $\beta$ and IL-18 from its proform $[46,47]$. IL-1 $\beta$ is a key inflammatory cytokine produced mainly by activated macrophages. Unlike most other cytokines, IL-1 $\beta$ maturation and release includes a twostep signal process. The first step is the synthesis of an inactive precursor form (pro-IL-1 $\beta$ ), which may be induced by the activation of NF- $\kappa B$ via Toll-like receptors. The second step is the maturation of IL-1 $\beta$, which depends on the activation of NLRP3 inflammasome. Excessive release of IL-1 $\beta$ upon the activation of NLRP3 inflammasome has been demonstrated to modulate liver inflammation and fibrosis $[10,12]$. IL-1 $\beta$ recruits other inflammatory cells, especially neutrophils, into the inflamed hepatic tissue leading to increased liver injury. The inhibition of IL-1 $\beta$ receptor has been shown to attenuate inflammasome-dependent alcoholic steatohepatitis [11, 48].

Our data show that NLRP3 inflammasome is activated in LCA-induced cholestatic liver injury. The mRNA and protein expression of NLRP3 inflammasome, caspase-1, and IL-1 $\beta$ were significantly increased in LCA group. Furthermore, the levels of caspase- 1 and IL-1 $\beta$ were significantly increased in hepatic tissue. IHC analysis of capase- 1 confirmed the PCR and western blot results. Our data are supported by a recent study by Gong et al [12]., which demonstrates the ability of chenodeoxycholic acid, a toxic bile acid, to induce NLRP3 inflammasome activation and subsequent secretion of the potent pro-inflammatory cytokine-IL-1 $\beta$, leading to cholestatic liver fibrosis. Importantly, vardenafil administration suppressed the activation of the NLRP3-caspase-1-IL-1 $\beta$ axis and provided a new insight into the hepatoprotective role of vardenafil.

Taken together, these data provided evidence that activation of the NLRP3-caspase-1-IL$1 \beta$ axis plays a critical role in the progression of cholestatic liver injury. Vardenafil treatment conferred protection against LCA-induced cholestatic liver damage through the activation of Nrf2 signaling and inhibition of NLRP3 inflammasome activation. Based on these findings, it is reasonable to suggest that vardenafil may be an appealing candidate for the treatment of cholestatic liver injury. 


\section{Cellular Physiology Cell Physiol Biochem 2018;47:523-534

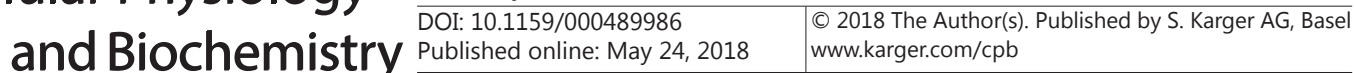 \\ El-Agamy et al.: Vardenafil and Cholestatic Liver}

\section{Abbreviations}

Nrf2 (nuclear factor-erythroid 2 related factor 2); GCLm (glutamate-cysteine ligase modifier); GCLc (glutamate-cysteine ligase catalytic subunit); Nqo1 (NAD(P)H quinone oxidoreductase 1); HO-1 (heme oxygenase-1); NLR (NOD-like receptor family); NLRP3 (NLR containing pyrin domain 3); ASC (apoptosis-associated speck-like protein containing a caspase activation and recruitment domain); IL-1 $\beta$ (interleukin-1 $\beta$ ); PDE-5 (phosphodiesterase type 5); LCA (lithocholic acid); ALT (alanine aminotransferase); AST (aspartate aminotransferase); ALP (alkaline phosphatase); LDH (lactate dehydrogenase); 4-HNE (4-hydroxynonenal); MDA (malondialdehyde); GSH (reduced glutathione); SOD (superoxide dismutase); TAC (total antioxidant capacity); NOx (nitrite/nitrate); NF-кB (nuclear factor kappa-B); TNF- $\alpha$ (tumor necrosis factor $\alpha$ ); IL-6 (interleukin-6).

\section{Acknowledgements}

The authors acknowledge the Deanship of Scientific Research and the Strategic Science Advanced Technology and Innovation Unit, Taibah University, for their assistance. The authors thank Mr. Mohamed Abdelsammad, College of Medicine, Taibah University, for his technical help.

DS El-Agamy was responsible for the conception and design of the study as well as writing the manuscript. DS El-Agamy and N Ahmed performed the experimental work. WD Alrohily, B Bojan, N Ahmed, MA Elkblawy, and HH Almaramhy performed the analytical measurements. MA Elkblawy performed the histopathology and IHC. The authors contributed equally to the acquisition of data and manuscript revision.

\section{Disclosure Statement}

The authors declare to have no competing interests.

\section{References}

1 Yang K, Kock K, Sedykh A, Tropsha A, Brouwer KL: An updated review on drug-induced cholestasis: mechanisms and investigation of physicochemical properties and pharmacokinetic parameters. J Pharm Sci 2013;102:3037-3057.

2 Kim MJ, Park SA, Kim CH, Park SY, Kim JS, Kim DK, Nam JS, Sheen YY: TGF- $\beta$ Type I Receptor Kinase inhibitor EW-7197 suppresses cholestatic liver fibrosis by inhibiting HIF1 $\alpha$ induced epithelial mesenchymal transition. Cell Physiol Biochem 2016;38:571-588.

-3 Xuan J, Wen W, Wang Y, Wang F, Xu HB, Shao M, Yang Y, Tian YZ: Effect of Huagantongluofang, a Chinese Traditional Medicine, in hepatic fibrogenesis in a mouse model of biliary cirrhosis. Cell Physiol Biochem 2017;44:368-376.

4 Ma X, Zhao YL, Zhu Y, Chen Z, Wang JB, Li RY, Chen C, Wei SZ, Li JY, Liu B, Wang RL, Li YG, Wang LF, Xiao XH: Paeonia lactiflora Pall. protects against ANIT-induced cholestasis by activating Nrf2 via PI3K/Akt signalling pathway. Drug Des Devel Ther 2015;9:5061-5074.

5 Yu L, Liu X, Yuan Z, Li X, Yang H, Yuan Z, Sun L, Zhang L, Jiang Z: SRT1720 alleviates ANITinduced cholestasis in a mouse model. Front Pharmacol 2017;8:256.

-6 Kensler TW, Wakabayashi N, Biswal S: Cell survival responses to environmental stresses via the Keap1Nrf2-ARE pathway. Annu Rev Pharmacol Toxicol 2007;47:89-116.

7 Shen K, Feng X, Pan H, Zhang F, Xie H, Zheng S: Baicalin ameliorates experimental liver cholestasis in mice by modulation of oxidative stress, inflammation and NRF2 transcription factor. Oxid Med Cell Longev 2017;2017:6169128.

8 Szabo G, Csak T: Inflammasomes in liver diseases. J Hepatol 2012;57:642-654.

$>9$ Dixon LJ, Berk M, Thapaliya S, Papouchado BG, Feldstein AE: Caspase-1-mediated regulation of fibrogenesis in diet-induced steatohepatitis. Lab Invest 2012;92:713-723. 


\section{Cellular Physiology Cell Physiol Biochem 2018;47:523-534

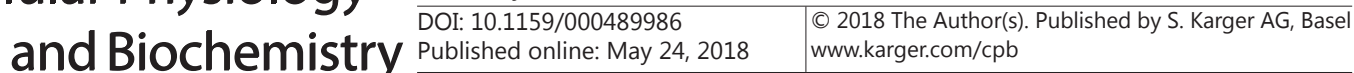

El-Agamy et al.: Vardenafil and Cholestatic Liver

10 Wree A, Eguchi A, McGeough MD, Pena CA, Johnson CD, Canbay A, Hoffman HM, Feldstein AE: NLRP3 inflammasome activation results in hepatocyte pyroptosis, liver inflammation, and fibrosis in mice. Hepatology 2014;59:898-910.

11 Gujral JS, Farhood A, Bajt ML, Jaeschke H: Neutrophils aggravate acute liver injury during obstructive cholestasis in bile duct-ligated mice. Hepatology 2003;38:355-363.

12 Gong Z, Zhou J, Zhao S, Tian C, Wang P, Xu C, Chen Y, Cai W, Wu J: Chenodeoxycholic acid activates NLRP3 inflammasome and contributes to cholestatic liver fibrosis. Oncotarget 2016;7:83951-83963.

13 Galie N, Ghofrani HA, Torbicki A, Barst RJ, Rubin LJ, Badesch D, Fleming T, Parpia T, Burgess G, Branzi A, Grimminger F, Kurzyna M, Simonneau G: Sildenafil use in pulmonary arterial hypertension (SUPER) study group, sildenafil citrate therapy for pulmonary arterial hypertension. N Engl J Med 2005;353:2148-2157.

14 Said E, Said SA, Gameil NM, Ammar EM: Modulation of thioacetamide-induced liver fibrosis/cirrhosis by sildenafil treatment. Can J Physiol Pharmacol 2013;91:1055-1063.

15 Savvanis S, Nastos C, Tasoulis MK, Papoutsidakis N, Demonakou M, Karmaniolou I, Arkadopoulos N, Smyrniotis V, Theodoraki K: Sildenafil attenuates hepatocellular injury after liver ischemia reperfusion in rats: a preliminary study. Oxid Med Cell Longev 2014;2014:161942.

16 Noel S, Panin N, Beka M, Dhooghe B, Huaux F, Leal T: Vardenafil reduces macrophage pro-inflammatory overresponses in cystic fibrosis through PDE5- and CFTR-dependent mechanisms. Clin Sci 2017;131:11071121.

17 Ahmed N, Bakhashwain NF, Alsehemi AF, El-Agamy DS: Hepatoprotective role of vardenafil against experimentally induced hepatitis in mice. J Biochem Mol Toxicol 2017;31(3).

18 Zhang X, Ma Z, Liang Q, Tang X, Hu D, Liu C, Tan H, Xiao C, Zhang B, Wang Y, Gao Y: Tanshinone IIA exerts protective effects in a LCA-induced cholestatic liver model associated with participation of pregnane $\mathrm{X}$ receptor. J Ethnopharmacol 2015;164:357-367.

19 Abo-Haded HM, Elkablawy MA, Al-Johani Z, Al-Ahmadi O, El-Agamy DS: Hepatoprotective effect of sitagliptin against methotrexate induced liver toxicity. PLoS One 2017;12:e0174295.

20 Hofmann AF: Detoxification of lithocholic acid, a toxic bile acid: relevance to drug hepatotoxicity. Drug Metab Rev 2004;36:703-722.

-21 Fickert P, Fuchsbichler A, Marschall HU, Wagner M, Zollner G, Krause R, Zatloukal K, Jaeschke H, Denk H, Trauner M: Lithocholic acid feeding induces segmental bile duct obstruction and destructive cholangitis in mice. Am J Pathol 2006;168:410-422.

22 Alashry SE, Gaballah MA, Malek HA, Abd Elsalam AI: Effect of sildenafil on non-alcoholic fatty liver. Int J Pharmacol 2015;11:814-820.

23 Aboutwerat A, Pemberton PW, Smith A, Burrows PC, McMahon RF, Jain SK, Warnes TW: Oxidant stress is a significant feature of primary biliary cirrhosis. Biochim Biophys Acta 2003;1637:142-150.

24 Radovits T, Arif R, Bömicke T, Korkmaz S, Barnucz E, Karck M, Merkely B, Szabó G: Vascular dysfunction induced by hypochlorite is improved by the selective phosphodiesterase-5inhibitor vardenafil. Eur J Pharmacol 2013;710:110-119.

-25 Yurtcu E, Togrul C, Ozyer S, Uzunlar O, Karatas YH, Seckin KD, Caydere M, Hucumenoglu $\mathrm{S}$, Cicek N: Dose dependent protective effects of vardenafil on ischemiareperfusion injury with biochemicaland histopathologic evaluation in rat ovary. J Pediatr Surg 2015;50:1205-1209.

26 Dias-Junior CA, Souza-Costa DC, Zerbini T, da Rocha JB, Gerlach RF, Tanus- Santos JE: The effect of sildenafil on pulmonary embolism-induced oxidative stress and pulmonary hypertension. Anesth Analg 2005;101:115-120.

27 Milani E, Nikfar S, Khorasani R, Zamani MJ, Abdollahi M: Reduction of diabetes-induced oxidative stress by phosphodiesterase inhibitors in rats. Comp Biochem Physiol C Toxicol Pharmacol 2005;140:251-255.

28 Hemnes AR, Zaiman A, Champion HC: PDE5A inhibition attenuates bleomycin-induced pulmonary fibrosis and pulmonary hypertension through inhibition of ROS generation and RhoA/Rho kinase activation. Am J Physiol Lung Cell Mol Physiol 2008;294:24-33.

-29 Semen K, Yelisyeyeva O, Jarocka-Karpowicz I, Kaminskyy D, Solovey L, Skrzydlewska E, Yavorskyi O: Sildenafil reduces signs of oxidative stress in pulmonary arterial hypertension: Evaluation by fatty acid composition, level of hydroxynonenal and heart rate variability. Redox Biol 2016;7:48-57. 


\section{Cellular Physiology Cell Physiol Biochem 2018;47:523-534

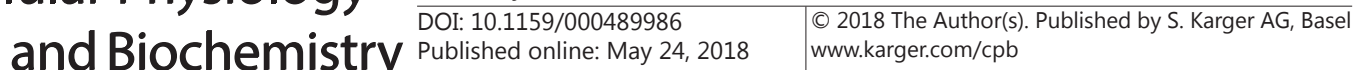

El-Agamy et al.: Vardenafil and Cholestatic Liver

30 Okada K, Shoda J, Taguchi K, Maher JM, Ishizaki K, Inoue Y, Ohtsuki M, Goto N, Sugimoto H, Utsunomiya H, Oda K, Warabi E, Ishii T, Yamamoto M: Nrf2 counteracts cholestatic liver injury via stimulation of hepatic defense systems. Biochem Biophys Res Commun 2009;389:431-436.

31 Wang T, Zhou ZX, Sun LX, Li X, Xu ZM, Chen M, Zhao GL, Jiang ZZ, Zhang LY: Resveratrol effectively attenuates a-naphthylisothiocyanate-induced acute cholestasis and liver injury through choleretic and anti-inflammatory mechanisms. Acta Pharmacol Sin 2014;35:1527-1536.

-32 Aleksunes LM, Slitt A L.,Maher J M, Dieter MZ, Knight TR, Goedken M, Cherrington NJ, Chan JY, Klaassen CD, Manautou JE: Nuclear factor-E2-related factor 2 expression in liver is critical for induction of NAD(P)H: quinone oxidoreductase 1 during cholestasis. Cell Stress Chaperones 2006;11:356-363.

-33 Tanaka Y, Aleksunes LM, Cui, YJ, Klaassen CD: ANIT-induced intrahepatic cholestasis alters hepatobiliary transporter expression via Nrf2- dependent and independent signalling. Toxicol Sci 2009;108:247-257.

34 Reisman SA, Aleksunes LM, Klaassen CD: Oleanolic acid activates Nrf2 and protects from acetaminophen hepatotoxicity via Nrf2-dependent and Nrf2-independent processes. Biochem Pharmacol 2009;77:12731282.

-35 Ben-Ari Z, Issan Y, Katz Y, Sultan M, Safran M, Michal LS, Nader GA, Kornowski R, Grief F, Pappo O, Hochhauser E: Induction of heme oxygenase 1 protects mouse liver from apoptotic ischemia/reperfusion injury. Apoptosis 2013;18:547-555.

-36 Garcia LA, Hlaing SM, Gutierrez RA, Sanchez MD, Kovanecz I, Artaza JN, Ferrini MG: Sildenafil attenuates inflammation and oxidative stress in pelvic ganglia neurons after bilateral cavernosal nerve damage. Int J Mol Sci 2014;15:17204-17220.

-37 Zahran MH, Hussein AM, Barakat N, Awadalla A, Khater S, Harraz A, Shokeir AA: Sildenafil activates antioxidant and antiapoptotic genes and inhibits proinflammatory cytokine genes in a rat model of renal ischemia/reperfusion injury. Int Urol Nephrol 2015;47:1907-1915.

38 Liu X-M, Peyton KJ, Durante W: Sildenafil stimulates heme oxygenase-1 gene expression in vascular smooth muscle cells via a soluble guanylate cyclase-independent pathway. FASEB J 2012;26:S1115.5.

39 Jin F, Cheng D, Tao JY, Zhang SL, Pang R, Guo YJ, Ye P, Dong JH, Zhao L: Anti-inflammatory and antioxidative effects of corilagin in a rat model of acute cholestasis. BMC Gastroenterol 2013;13:79.

40 Gäbele E, Froh M, Arteel GE, Uesugi T, Hellerbrand C, Schölmerich J, Brenner DA, Thurman RG, Rippe RA: TNF $\alpha$ is required for cholestasis-induced liver fibrosis in the mouse. Biochem Biophys Res Commun 2009;378:348-353.

41 Gowert NS, Klier M, Reich M, Reusswig F, Donner L, Keitel V, Häussinger D, Elvers M: Defective platelet activation and bleeding complications upon cholestasis in mice. Cell Physiol Biochem 2017;41:2133-2149.

42 Lubamba B, Huaux F, Lebacq J, Marbaix E, Dhooghe B, Panin N, Wallemacq P, Leal T: Immunomodulatory activity of vardenafil on induced lung inflammation in cystic fibrosis mice. J Cyst Fibros 2012;11:266-273.

43 Zhou R, Tardivel A, Thorens B, Choi I, Tschopp J: Thioredoxin-interacting protein links oxidative stress to inflammasome activation. Nat Immunol 2010;11:136-140.

-44 Dostert C, Petrilli V, Van Bruggen R, Steele C, Mossman BT, Tschopp J: Innate immune activation through Nalp3 inflammasome sensing of asbestos and silica. Science 2008;320:674-677.

45 Muruve DA, Petrilli V, Zaiss AK, White LR, Clark SA, Ross PJ, Parks RJ, Tschopp J: The inflammasome recognizes cytosolic microbial and host DNA and triggers an innate immune response. Nature 2008;452:103-107.

-46 Liu RT, Gao J, Cao S, Sandhu N, Cui JZ, Chou CL, Fang E, Matsubara JA: Inflammatory mediators induced by amyloid-beta in the retina and RPE in vivo: implications for inflammasome activation in age-related macular degeneration. Invest Ophthalmol Vis Sci 2013;54:2225-2237.

47 Lei C, Lin R, Wang J, Tao L, Fu X, Qiu Y, Lei B: Amelioration of amyloid $\beta$-induced retinal inflammatory responses by a LXR agonist T0901317 is associated with inhibition of the NF- $\mathrm{KB}$ signaling and NLRP3 inflammasome. Neuroscience 2017;360:48-60.

48 Petrasek J, Bala S, Csak T, Lippai D, Kodys K, Menashy V, Barrieau M, Min SY, Kurt-Jones EA, Szabo G: IL-1 receptor antagonist ameliorates inflammasome dependent alcoholic steatohepatitis in mice. J Clin Invest 2012;122:3476-3489. 